\title{
The Wide Field Imager Lyman-Alpha Search (WFILAS) for galaxies at redshift $\sim 5.7^{\star}$
}

\section{A spatially compact Ly $\alpha$ emitting galaxy at redshift $\mathbf{5 . 7 2 1}$}

\author{
E. Westra ${ }^{1}$, D. Heath Jones ${ }^{1}$, C. E. Lidman ${ }^{2}$, R. M. Athreya ${ }^{3}$, K. Meisenheimer ${ }^{4}$, C. Wolf $^{5}$, T. Szeifert ${ }^{2}$, \\ E. Pompei ${ }^{2}$, and L. Vanzi ${ }^{2}$ \\ ${ }^{1}$ Research School of Astronomy \& Astrophysics, The Australian National University, Weston Creek ACT 2611, Australia \\ e-mail: [westra; heath] @mso.anu.edu.au \\ 2 European Southern Observatory, Casilla 19001, Santiago 19, Chile \\ e-mail: [clidman; tszeifer; epompei;lvanzi]@eso.org \\ ${ }^{3}$ National Centre for Radio Astrophysics, Tata Institute of Fundamental Research Pune University Campus, Post Bag 3 , \\ Ganeshkhind Pune 411007, India \\ e-mail: rathreya@ncra.tifr.res.in \\ ${ }^{4}$ Max Planck Institute für Astronomie, Königstuhl 17, 69117 Heidelberg, Germany \\ e-mail: meise@mpia.de \\ 5 Department of Astrophysics, Denys Wilkinson Building, University of Oxford, Keble Road, Oxford, OX1 3RH, UK \\ e-mail: cwolf@astro.ox.ac.uk
}

Received 1 October 2004 / Accepted 26 November 2004

\begin{abstract}
We report the spectroscopic confirmation of a compact Ly $\alpha$ emitting galaxy at $z=5.721$. A FORS 2 spectrum of the source shows a strong asymmetric line with a flux of $5 \times 10^{-17} \mathrm{erg} \mathrm{s}^{-1} \mathrm{~cm}^{-2}$, making it one of the brightest Ly $\alpha$ emitting galaxies at this redshift, and a line-of-sight velocity dispersion of $400 \mathrm{~km} \mathrm{~s}^{-1}$. We also have a tentative detection of a second, narrower component that is redshifted by $400 \mathrm{~km} \mathrm{~s}^{-1}$ with respect to the main peak. A FORS2 image shows that the source is compact, with a $F W H M$ of 0.5 , which corresponds to $3.2 \mathrm{kpc}$ at this redshift ${ }^{\star \star}$. This source is a brighter example of J1236.8+6215 (Dawson et al. 2002, ApJ, 570, 92), another Ly $\alpha$ emitting galaxy at $z \sim 5.2$.
\end{abstract}

Key words. galaxies: high-redshift - galaxies: evolution - galaxies: starburst - galaxies: individual: J114334.98-014433.9

\section{Introduction}

Wide-field imaging surveys with specially selected narrowband filters are an effective means of discovering high redshift $(z \gtrsim 5)$ Ly $\alpha$ emitting galaxies (see Hu et al. 2004, and references therein). Spectra of these galaxies are dominated by a single, asymmetric emission line. One of the strongest arguments for associating this line with Lyman- $\alpha(\operatorname{Ly} \alpha)$ is the asymmetry in the line profile (e.g. Stern et al. 2000), which can only be detected if the spectral resolution is high enough $(R \gtrsim 2000)$.

The profile of the Ly $\alpha$ line is the end result of emission from HII regions and resonant scattering by HI. The bulk of the Ly $\alpha$ emission comes from the recombination of hydrogen that has been ionised by UV flux of massive stars. Part of the

^ Based on observations taken at the Cerro La Silla (ESO programs 67.A-0063, 68.A-0363, 69.A-0314 and MPG time) and Cerro Paranal Observatories (ESO program 272.A-5029).

$\star \star$ Throughout this letter, a cosmology with $H_{0}=70 \mathrm{~km} \mathrm{~s}^{-1} \mathrm{Mpc}^{-1}$, $\Omega_{\mathrm{M}}=0.3$ and $\Omega_{\Lambda}=0.7$ is assumed. Magnitudes are on the AB-system. ionisation may be due to shocks (Bland-Hawthorn \& Nulsen 2004) or from an AGN, although in a study of Ly $\alpha$ emitting galaxies at $z \sim 4.5$, Wang et al. (2004) found no evidence of AGN activity. The shape of the line profile is sensitive to the geometry, density and kinematics of both the HII gas, where the line is produced, and the HI gas, where it is scattered (Ahn et al. 2003, Santos et al. 2004). Dust can also play a role. Hence, the Ly $\alpha$ emission line can be viewed as a tool, albeit a rather blunt one, that might be used to constrain the spatial and kinematic distribution of the hydrogen gas in these distant galaxies.

In this letter, we present the 0.5 resolution seeing-limited imaging and $R \sim 3600$ spectroscopy of a Ly $\alpha$ emitting galaxy that was selected from Ly $\alpha$ emitting candidates in the WFILAS catalog (Westra et al. 2005, in prep.).

\section{WFILAS and candidate selection}

WFILAS is a survey for bright $\operatorname{Ly} \alpha$ emitting galaxies at $z \sim 5.7$. The selection strategy is similar to the successful strategies employed in other Ly $\alpha$ surveys at these redshifts 


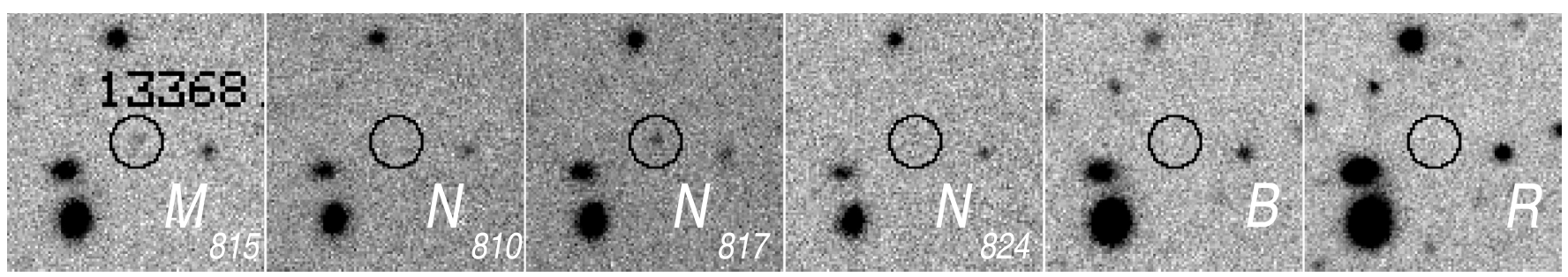

Fig. 1. From left to right, thumbnails of the confirmed Ly $\alpha$ emitter S11_13368 at redshift 5.721. Each thumbnail covers a $24^{\prime \prime} \times 24^{\prime \prime}$ region with a pixel scale of $0.238 \mathrm{pix}^{-1}$. The emitter is only detected in the $M_{815}$ and $N_{817}$ images.

(Ajiki et al. 2003; Rhoads et al. 2003; Hu et al. 2004). WFILAS covers a larger volume $\left(\sim 1.2 \times 10^{6} \mathrm{Mpc}^{3}\right)$, a larger area $\left(\sim 1 \square^{\circ}\right)$ and has a brighter detection limit ( $2 \sigma$ limit magnitude $\sim 24.0$ 24.5) than these surveys. Hence the candidate Ly $\alpha$ emitting galaxies in the WFILAS catalogue will, on average, be brighter than the Ly $\alpha$ emitting galaxies in these other catalogs.

The survey used the Wide Field Imager (WFI) on the ESO/MPI $2.2 \mathrm{~m}$ telescope at the Cerro La Silla Observatory and targeted three fields. The WFI consists of a mosaic of eight $(4 \times 2) 2 \mathrm{k} \times 4 \mathrm{k}$ CCDs arranged to give a field of view of $34^{\prime} \times 33^{\prime}$ with a pixel scale of $0^{\prime} \cdot 238$ pixel $^{-1}$. Images were taken with the standard broad-band $B$ and $R$ filters, and four narrower filters - an intermediate-band $(\Delta \lambda=22 \AA)$ filter at $815 \mathrm{~nm}\left(M_{815}\right)$ and three custom-made narrow-band $(\Delta \lambda=7 \AA)$ filters with central wavelengths at $810 \mathrm{~nm}\left(N_{810}\right), 817 \mathrm{~nm}\left(N_{817}\right)$ and $824 \mathrm{~nm}\left(N_{824}\right)$. They lie in a spectral region where the emissivity of the night-sky is relatively low, which improves the sensitivity to $\operatorname{Ly} \alpha$ emission.

Candidate Ly $\alpha$ galaxies are those that appear in one of the narrow band filters, but are undetected in the broad band filters. Given the relatively low signal-to-noise ratios of the candidates, we also require a detection in the intermediate band filter. This limits the number of spurious candidates. We refer the interested reader to Westra et al. (2005, in prep.) for a description of the observations, reduction and candidate selection.

\section{Confirmed Ly $\alpha$ emitter at $z=5.721$}

In a pilot study to test the effectiveness of the selection strategy one of the brighter candidates (J114334.98-014433.9, hereafter S11_13368) was observed with FORS2 on Yepun (UT4) at the Cerro Paranal Observatory (Fig. 1).

A pre-image with an intermediate-band filter $(13 \mathrm{~nm})$ centered at $815 \mathrm{~nm}$ was taken with FORS2 on 2004 February 16th in which S11_13368 clearly was detected. Figure 2 shows a 30" region around S11_13368. The FWHM of stars in this field are 0'.5 and S11_13368 is unresolved.

Three $1200 \mathrm{~s}$ exposures were taken on 2004 March 18th with FORS2 using the $1028 \mathrm{z}$ grism and a $1^{\prime \prime}$ slit. Frames were bias-subtracted and flatfielded and were then combined with suitable pixel rejection to remove cosmic rays. The 2D-spectrum (without subtracting the sky lines) and the extracted sky-subtracted spectrum are shown in Figs. 3a,b, where one can clearly see a single emission line with a broad red wing. No continuum is detected, implying a $2 \sigma$ upper-limit for the continuum of $7 \times 10^{-20} \mathrm{erg} \mathrm{s}^{-1} \mathrm{~cm}^{-2} \AA^{-1}$ over the rest frame wavelength range 1220 to $1230 \AA$.

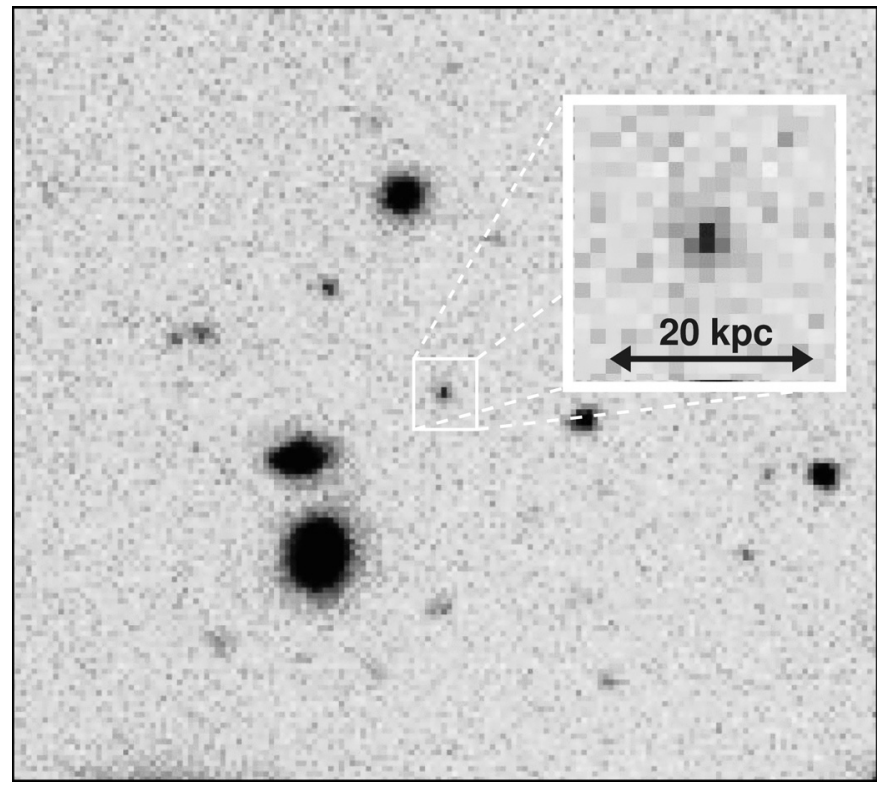

Fig. 2. A $30^{\prime \prime} \times 30^{\prime \prime}$ region around the confirmed $\operatorname{Ly} \alpha$ galaxy S11_13368 at $z=5.721$ from the pre-image taken with FORS2. The image has a pixel scale of $0.252 \mathrm{pix}^{-1}$. The object is unresolved in this image. The seeing at the time of these observations was $\sim 0$ ' 5 . The exposure time was $3600 \mathrm{~s}$.

Stern et al. (2000) have reviewed the different ways in which high redshift Ly $\alpha$ can be verified and suggest that line asymmetry is the surest way. Our spectral resolution $(R \sim 3600)$ is high enough to securely confirm this asymmetry. This resolving power is also more than adequate to rule out [O II] $\lambda \lambda 3726,3728$ at $z=1.19$, since we do not resolve the line into the close doublet. The separation of this doublet at $z=1.19$ is $6.1 \AA$ and therefore easily resolvable. Similarly, we can rule out the possibility that the line is [O III] $\lambda \lambda 4959,5007$ at $z=0.63$, due to the absence of the accompanying line in that doublet. We can also rule out $\mathrm{H} \alpha$ at $z=0.25$. If the line was $\mathrm{H} \alpha$, then we should have either detected [N II] and/or some flux in the $R$-band due to the contributions of $\mathrm{H} \beta$, [O III] $\lambda \lambda 4959,5007$ and the continuum (e.g. Kniazev et al. 2004). In Fig. 1, one can see that there is no detection in the $R$-band image. Given the absence of all of these potential neighbouring features, and also the clear asymmetry of the line, we identify it as $\operatorname{Ly} \alpha$ emission at $z=5.721$.

The integrated line flux of the line derived from the spectrum is $5 \times 10^{-17} \mathrm{erg} \mathrm{s}^{-1} \mathrm{~cm}^{-2}$, making it one of the brightest Ly $\alpha$ emitting sources at this redshift (cf. Ajiki et al. 2003; 


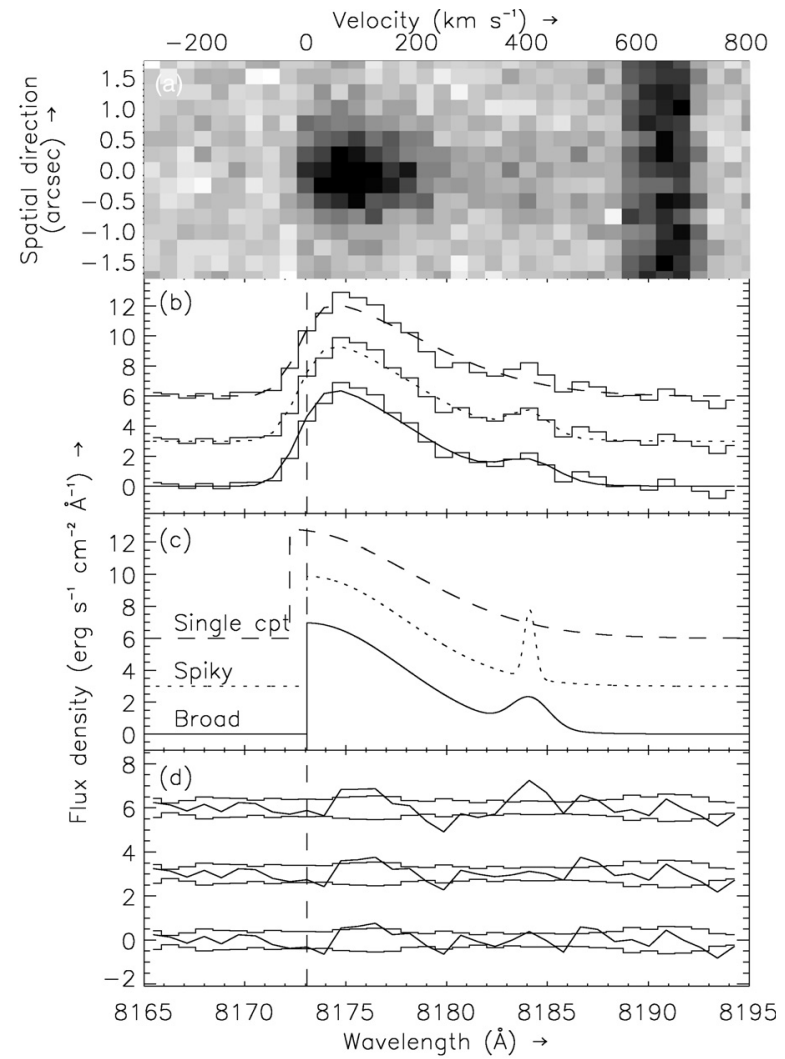

Fig. 3. A fit of both one- and two-component models to the profile of the Ly $\alpha$ line. a) The two dimensional spectrum centered on the Ly $\alpha$ line. In this unrectified spectrum, the night sky emission lines are not removed. The pixel scale for this image is $0.252 \mathrm{pix}^{-1}$ in the spatial direction and $0.86 \AA_{\mathrm{pix}^{-1}}$ in the dispersion direction. b) Observed Ly $\alpha$ line (histograms) with the three best-fitting models. For clarity, the models are offset by $3 \mathrm{erg} \mathrm{s}^{-1} \mathrm{~cm}^{-2} \AA^{-1}$. The twocomponent models consist of a two components: a broad, truncated Gaussian and a narrower redshifted Gaussian. The one-component model consists of only a broad, truncated Gaussian. See Table 1 for the parameters of the "broad" (solid), "spiky" (dotted) and "single component" (dashed) models. c) Same model line profiles as in b) but before convolution with the instrument profile. d) Observed data minus model fit (as plotted in b)) residuals, demonstrating a random scatter about the zero flux line. Also shown (histograms) is the $1 \sigma$ error spectrum from the observed data, which includes both sky- and Poisson noise. Note that the red peak is not N V. At this redshift it would appear around $8334 \AA$.

Rhoads et al. 2003; Maier et al. 2003). The $2 \sigma$ lower limit on the rest frame equivalent width is $\sim 100 \AA$. At $z=5.721$, this translates to a Ly $\alpha$ luminosity of $1.8 \times 10^{43} \mathrm{erg} \mathrm{s}^{-1}$ suggesting an apparent star-formation rate of $16 M_{\odot} \mathrm{yr}^{-1}$, using the conversion rate from Ajiki et al. (2003).

Following earlier works (Dawson et al. 2002; Hu et al. 2004), we fit both two- and single-component models to the $\operatorname{Ly} \alpha$ line. The two-component fit consists of a truncated Gaussian with complete absorption bluewards of $\operatorname{Ly} \alpha$ line center and a redshifted Gaussian that is not truncated (e.g. Hu et al. 2004). The one-component fit consists solely of a truncated Gaussian (Figs. 3b and c). Since the seeing was narrower than the width of the slit, we convolve the model with a Gaussian that has a $F W H M$ of $2.3 \AA$. At $8175 \AA$ this corresponds to a
Table 1. Model fit parameters as described in Sect. 3 and indicated in Fig. 3.

\begin{tabular}{ccccccc}
\hline \hline Component & $\lambda_{c}$ & $f_{\text {peak }}$ & \multicolumn{2}{c}{$F W H M$} & \multicolumn{3}{c}{$\Delta v$} \\
$(1)$ & $(2)$ & $(3)$ & $(4)$ & $(5)$ & $(6)$ & $(7)$ \\
\hline \multicolumn{7}{c}{ "Broad" model } \\
Main peak & 8173.1 & 7.0 & 11.1 & 408 & $\ldots$ & $\ldots$ \\
Red peak & 8184.2 & 1.9 & 2.2 & 81 & +11.1 & 406 \\
\hline \multicolumn{7}{c}{ "Spiky" model } \\
Main peak & 8173.1 & 6.9 & 11.4 & 419 & $\ldots$ & $\ldots$ \\
Red peak & 8184.1 & 4.3 & 0.6 & 24 & +11.0 & 405 \\
\hline \multicolumn{7}{c}{ "Single component" model } \\
Main peak & 8172.2 & 6.8 & 14.0 & 514 & $\ldots$ & $\ldots$ \\
\hline
\end{tabular}

Notes: (1) component of the fit, (2) central wavelength of the fitted component in $\AA$, (3) peak flux density in $10^{-18} \mathrm{erg} \mathrm{s}^{-1} \mathrm{~cm}^{-2} \AA^{-1}$, (4) and (5) $F W H M$ of full Gaussian of the profile in $\AA$ and $\mathrm{km} \mathrm{s}^{-1}$, respectively, (6) and (7) line-of-sight outflow velocity in $\AA$ and $\mathrm{km} \mathrm{s}^{-1}$, respectively.

resolution of $R \sim 3600$. We use the Levenberg-Marquardt nonlinear least-squares algorithm to find the best fit.

Two different two-component models fit the data with a similar reduced $\chi_{v}^{2}$ values of $\sim 1.3$. We refer to these two models as the "broad" model and the "spiky" model. In the "broad" model, the redshifted component is broader and weaker and the central component is narrower and stronger in comparison to the "spiky" model. After convolving with the instrumental profile both fits have similar residuals. Both models have a central peak at a wavelength corresponding to $\operatorname{Ly} \alpha$ at $z=5.721$. The redshifted component is clearly detected in both models and lies $\sim+400 \mathrm{~km} \mathrm{~s}^{-1}$ away from the central peak. Given the similar reduced $\chi_{v}^{2}$, we cannot favour one model over the other.

The single-component model has a broader main peak, which is slightly bluer. This model does not fit the profile as well as the two-component cases, particularly in the region of the red peak $\left(\chi_{v}^{2}=2.2\right)$.

Table 1 summarises the different model components.

The integrated line fluxes for the two-component models are very similar. If one were to include the flux that was missing from the blue side of the truncated Gaussian, the total a line flux is $8.3 \times 10^{-17} \mathrm{erg} \mathrm{s}^{-1} \mathrm{~cm}^{-2}$. This corresponds to a star-formation rate of $\sim 27 M_{\odot} \mathrm{yr}^{-1}$, using the conversion rate from Ajiki et al. (2003). The width on the blueward side of the profile is solely due to instrumental broadening.

\section{Discussion}

We have presented a medium resolution spectrum of a bright Ly $\alpha$ emitting galaxy at $z=5.721$. The spectrum consists of a single emission line and no continuum. The line shows a distinct asymmetry, which undoubtedly confirms it as Ly $\alpha$. We model the line with two components: a one-sided Gaussian and a narrower, redshifted component. The profile of the blue side of the line is entirely defined by the instrument profile.

Generally, the second component is less frequently observed, although it is possible that it has been missed in the spectra of other Ly $\alpha$ emitting galaxies. Most of these spectra were taken at lower resolution and are considerably noisier. 


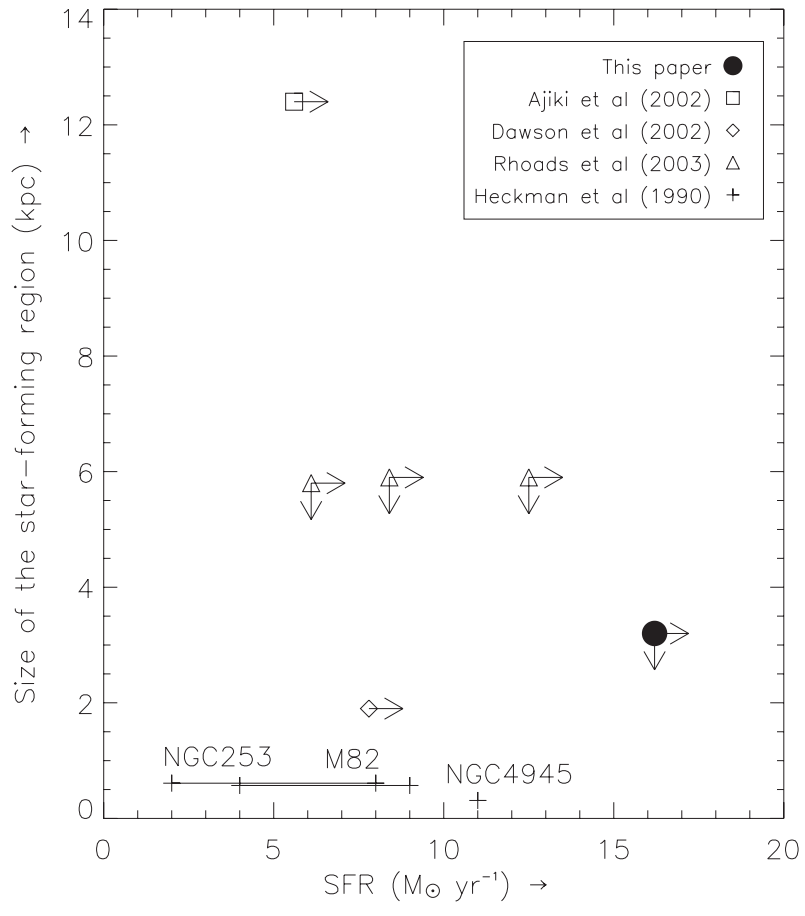

Fig. 4. Size of the star-forming region versus the star-formation rate. The SFR of two galaxies from Heckman et al. (1990) is indicated by a bar, as there are two measurements of the SFR. The arrows represent lower-limits to the SFR and upper-limits to the size of the major axis, (due to seeing limited observations). Error-bars are not included. The plus signs represent three well-known local starbursting galaxies (Heckman et al. 1990).

A second peak in the Ly $\alpha$ line is a clear signature of an expanding shell of neutral hydrogen (Dawson et al. 2002; Ahn et al. 2003). The strength and shape of the secondary peak depends on the kinematics and the quantity of neutral hydrogen in the expanding shell and the amount and distribution of dust throughout the galaxy (Ahn 2004).

S11_13368 appears to be a brighter and more distant example of J1235.8+6215 at $z=5.190$ (Dawson et al. 2002). The line profile is strongly asymmetric in both objects, and both suggest a second redshifted component. Both objects are also very compact. However, there are some noteworthy differences. The Ly $\alpha$ line in S11_13368 is considerably broader, and the redshifted component is a lot narrower, even in our "broad" model.

The intrinsic Ly $\alpha$ profile is heavily modified by the surrounding gas and the fraction of the line that is finally observed is very model dependent (Santos 2004; Ahn 2004). In general, it is only a fraction of the intrinsic flux. Hence, star formation rates that are estimated from the observed Ly $\alpha$ flux directly, as they are done in this paper, could drastically underestimate the true star formation rate. Similarly, the centroid of the observed profile is also model dependent. This directly leads to an uncertainty in the redshift of about 0.01 , if no other lines are visible, which is usually the case for such high redshift galaxies.
S11_13368, like J1235.8+6215, is very compact. With a projected size of $\sim 3 \mathrm{kpc}$ or less, it is comparable to the size of the star forming regions in local starbursting galaxies; however, the star formation rate is much higher. Not all Ly $\alpha$ emitting galaxies at $z \sim 5.7$ are as compact. In Fig. 5, we plot apparent size of the star forming region versus the inferred star formation rate for a sample of local starbursts and distant galaxies. The emission line region in LAE J1044-0130 occurs over a region that is an order of magnitude larger than emission line regions in S11_13368 and J1235.8+6215 even though the inferred star formation rate is significantly less. Given that the projected star formation rate per unit area in S11_13368 far exceeds $0.1 M_{\odot} \mathrm{yr}^{-1} \mathrm{kpc}^{-2}$, it is likely that a hot, enriched starburst-driven gas is outflowing into the halo of S11_13368, facilitating the enrichment of the halo and the escape of Lyman continuum photons (Heckman et al. 2000; Tenorio-Tagle et al. 1999).

Acknowledgements. The authors wish to thank the Max-Planck-Institut für Astronomie and the DDT grant of the European Southern Observatory for providing the narrow band filters which are crucial to the WFILAS survey. We also like to thank the anonymous referee for his/her useful suggestions and comments, which made us improve the article a lot. D. H. Jones is supported as a Research Associate by Australian Research Council DiscoveryProjects Grant (DP-0208876), administered by the Australian National University. C. Wolf was supported by a PPARC Advanced Fellowship. Reduction was done with IRAF, which is distributed by the National Optical Astronomy Observatories, which are operated by the Association of Universities for Research in Astronomy, Inc., under cooperative agreement with the National Science Foundation.

\section{References}

Ahn, S. 2004, ApJ, 601, L25

Ahn, S., Lee, H., \& Lee, H. M. 2003, MNRAS, 340, 863

Ajiki, M., Taniguchi, Y., Fujita, S. S., et al. 2003, AJ, 126, 2091

Bland-Hawthorn, J. \& Nulsen, P. E. J. 2004

[arXiv: astro-ph/0404241]

Dawson, S., Spinrad, H., Stern, D., et al. 2002, ApJ, 570, 92

Heckman, T. M., Armus, L., \& Miley, G. K. 1990, ApJS, 74, 833

Heckman, T. M., Lehnert, M. D., Strickland, D. K., \& Armus, L. 2000, ApJS, 129, 493

Hu, E. M., Cowie, L. L., Capak, P., et al. 2004, AJ, 127, 563

Kniazev, A. Y., Pustilnik, S. A., Grebel, E. K., Lee, H., \& Pramskij, A. G. 2004, ApJS, 153, 429

Maier, C., Meisenheimer, K., Thommes, E., et al. 2003, A\&A, 402, 79

Rhoads, J. E., Dey, A., Malhotra, S., et al. 2003, AJ, 125, 1006

Santos, M. R. 2004, MNRAS, 349, 1137

Santos, M. R., Ellis, R. S., Kneib, J., Richard, J., \& Kuijken, K. 2004, ApJ, 606, 683

Stern, D., Bunker, A., Spinrad, H., \& Dey, A. 2000, ApJ, 537, 73

Tenorio-Tagle, G., Silich, S. A., Kunth, D., Terlevich, E., \& Terlevich, R. 1999, MNRAS, 309, 332

Wang, J. X., Rhoads, J. E., Malhotra, S., et al. 2004, ApJ, 608, L21 